\title{
Spatially modulated polarimetry based on a vortex retarder and Fourier analysis
}

\author{
Chao Gao (高 超) and Bing Lei (雷 兵) \\ College of Advanced Interdisciplinary Studies, National University of Defense Technology, Changsha 410073, China \\ *Corresponding author: cslb2017@163.com \\ Received July 20, 2020 | Accepted September 4, 2020 | Posted Online December 3, 2020
}

\begin{abstract}
We report a spatially modulated polarimetry scheme by using a zero-order vortex half-wave retarder (ZVHR) and a spatial Fourier analysis method. A ZVHR is employed to analyze the input polarized light and convert it into a vectorial optical field, and an analyzer is set after the ZVHR to form an hourglass intensity pattern due to the spatial polarization modulation. Then, the input light's Stokes parameters can be calculated by spatial Fourier analysis of the hourglass pattern with a single shot. The working principle of the polarimeter has been analyzed by the Stokes-Mueller formalism, and some quantitative measuring experiments of different polarization states have been demonstrated. The experimental results indicate that the proposed polarimeter is accurate, robust, and simple to use.
\end{abstract}

Keywords: polarimetry; Stokes parameters; vortex retarder; Fourier analysis; spatial modulation.

DOI: 10.3788/COL202119.021201

\section{Introduction}

Polarization is an essential property of light, and measuring the polarization state fast and precisely is an important issue in many scientific research fields, such as ellipsometry ${ }^{[1]}$, remote sensing ${ }^{[2]}$, optical communication ${ }^{[3]}$, and biomedicine ${ }^{[4]}$. One conventional way for polarimetry is to measure the intensity change when the polarized beam is modulated by rotating optical components ${ }^{[5,6]}$, and the Stokes parameters can be obtained by analyzing the temporally varying intensity. Another polarimetric way is to split the input beam into several channels, and its polarization state can be obtained by analyzing each channel with different polarization optics ${ }^{[7]}$. Unfortunately, the method of rotating optical components is not stable, and it is only suitable for static or slowly varying processes. The multi-channel scheme is competent for real-time monitoring, but it is usually sensitive to the fluctuations of the optical source's wavelength and output power, and, moreover, its configuration is usually complicated and difficult to adjust ${ }^{[8,9]}$.

To overcome the adverse effects of conventional methods and to satisfy the increasing demands for fast and precise measurement, researchers have proposed many new polarimetric schemes, such as a liquid crystal retarder (LCR) polarimeter $^{[10,11]}$, interferometric polarimeter ${ }^{[12-14]}$, metasurface-based polarimeter $^{[15-17]}$, and spatially modulated polarimeter ${ }^{[18,19]}$. The spatially modulated scheme may be an easy and efficient method in polarimetric measurement, which has the potential to simplify system configuration and decrease the measurement time. Many spatially modulated polarimeters have been achieved by using various optical components, including polarization gratings ${ }^{[20-22]}$, Savart polariscopes ${ }^{[23,24]}$, and birefringent prisms ${ }^{[25,26]}$. However, few study has adequately explored the possibility that the vortex retarder ${ }^{[27]}$ can be a very simple and efficient optical device to realize spatial modulation in polarimetry.

The vortex retarder is a special wave plate that has a constant retardance across the clear aperture, but its fast axis rotates continuously along the azimuth ${ }^{[27]}$, so an ordinary polarized light can be converted into a vectorial optical beam ${ }^{[28,29]}$ by the vortex retarder. The spatial inhomogeneous polarization distribution of the vectorial optical beam provides the possibility for spatial modulation, rather than temporal modulation in traditional polarimetry, which can be helpful to achieve better stability and less measurement time.

In our earlier research, a spatially modulated ellipsometric scheme by using a vortex retarder has been demonstrated theoretically ${ }^{[30]}$, but the measurement result was extracted from two intensity images, and the optical components also needed to be rotated, which limited the measurement speed and stability severely. In this Letter, we explored the possibility of the vortex retarder for polarimetric measurement and experimentally demonstrated a spatially modulated polarimetry scheme based on a vortex retarder and a spatial Fourier analysis method, which can achieve simultaneous measurement of the Stokes parameters at a single shot. 


\section{Theoretical and Experimental Investigation}

The schematic of the experimental setup is shown in Fig. 1, which includes two parts, the polarization state generation (PSG) system and the polarization state detection (PSD) system. In the PSG system, an ordinary He-Ne laser with the wavelength of $632.8 \mathrm{~nm}$ was employed as an optical source, and an integrating sphere and a positive lens (focal length $100 \mathrm{~mm}$ ) were utilized to produce a uniform and nearly collimated light field for spatial polarization modulation. The incident beams with different states of polarization (SOPs) were generated by a linear polarizer (Thorlabs, LPVISE100-A) and a quarter-wave plate (Thorlabs, WPQ10E-633), which were mounted on the motorized rotation stages (Thorlabs, KPRM1E). In the PSD system, the zero-order vortex half-wave retarder (ZVHR) (Thorlabs, WPV10L-633) was utilized to convert the unknown input polarized light into a vectorial optical field, and an hourglassshaped intensity distribution can be obtained when this vectorial optical field is analyzed by a linear polarizer (Thorlabs, LPVISE100-A $)^{[30,31]}$. A scientific complementary metal-oxidesemiconductor (CMOS) camera (Tucsen, Dhyana95) was utilized to record the intensity distribution, and then the Stokes parameters of the input light can be obtained based on the spatial Fourier analysis of the hourglass intensity pattern.

The operating principle of our proposed polarimeter (PSD system) can be analyzed with the Mueller matrix formula as

$$
S^{\prime}=M_{A} \cdot M_{\mathrm{ZVHR}} \cdot S,
$$

where $S=\left[\begin{array}{llll}S_{0} & S_{1} & S_{2} & S_{3}\end{array}\right]^{T}, S^{\prime}=\left[\begin{array}{llll}S_{0}^{\prime} & S_{1}^{\prime} & S_{2}^{\prime} & S_{3}^{\prime}\end{array}\right]^{T}$ are the Stokes vectors of the input and output light; $M_{\mathrm{ZVHR}}$ and $M_{A}$ are the Muller matrix of the ZVHR and the analyzer. In the PSD system, the initial fast axis of the ZVHR and the transmission axis of the analyzer are oriented at $0^{\circ}$, and $M_{\mathrm{ZVHR}}$ is given as

$$
M_{\mathrm{ZVHR}}=\left[\begin{array}{cccc}
1 & 0 & 0 & 0 \\
0 & \cos 2 \varphi & \sin 2 \varphi & 0 \\
0 & \sin 2 \varphi & -\cos 2 \varphi & 0 \\
0 & 0 & 0 & -1
\end{array}\right],
$$

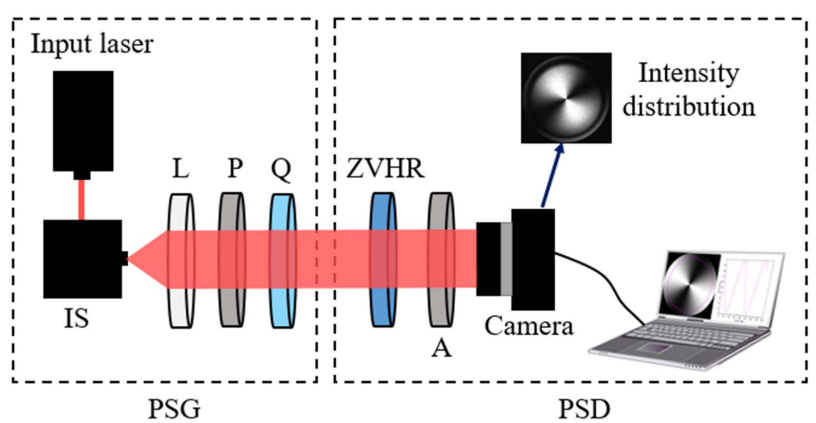

Fig. 1. Schematic of our experimental configuration. IS, integrating sphere; L, positive lens; $P$, polarizer; $Q$, quarter-wave plate; ZVHR, zero-order vortex halfwave retarder; $A$, analyzer where the variable parameter $\varphi$ is the azimuth angle. The intensity $I(\varphi)$ recorded by the camera is proportional to $S_{0}^{\prime}$ and can be given as

$$
I(\varphi) \propto S_{0}^{\prime}=S_{0}+S_{1} \cos 2 \varphi+S_{2} \sin 2 \varphi .
$$

Equation (3) represents a truncated spatial Fourier series whose coefficients are determined by the Stokes parameters of the incident polarized beam ${ }^{[19,22]}$, and these parameters can be obtained by the spatial Fourier analysis of the outcome intensity and described as

$$
\left\{\begin{array}{l}
S_{0}=\frac{1}{2 \pi} \int_{-\pi}^{\pi} I(\varphi) \mathrm{d} \varphi \\
S_{1}=\frac{1}{\pi} \int_{-\pi}^{\pi} I(\varphi) \cos 2 \varphi \mathrm{d} \varphi . \\
S_{2}=\frac{1}{\pi} \int_{-\pi}^{\pi} I(\varphi) \sin 2 \varphi \mathrm{d} \varphi
\end{array}\right.
$$

If the first Stokes parameter is normalized (i.e., $S_{0}=1$ ), and $S_{1}$ and $S_{2}$ are also normalized with respect to $S_{0}$, then the fourth Stokes parameter $S_{3}$ can be calculated as

$$
S_{3}=\sqrt{\mathrm{DOP}^{2}-S_{1}^{2}-S_{2}^{2}},
$$

where the DOP is the degree of polarization of the input light.

However, the measured accuracy of Stokes parameters will be impaired by many factors in real experiments, such as ambient stray light, imperfections of optical components, and image noise. The comprehensive effect of these adverse factors will cause a decrease of the image contrast, even though the incident light of the polarimeter is a fully polarized light, and the obtained spatially modulated image will be similar to the situation where a partially polarized light is input into the polarimeter. Therefore, the measurement errors caused by the above adverse factors can be calibrated by attributing them to the decrease of DOP, and the actual DOP can be estimated by employing a typical linearly polarized light with known polarization state as a probe light. Since the parameters $S_{0}, S_{1}$, and $S_{2}$ can be calculated by the proposed scheme directly, the DOP of a linearly polarized probe light can be obtained as

$$
\mathrm{DOP}=\sqrt{S_{1}^{2}+S_{2}^{2}}
$$

Before the formal measurements, an arbitrary linearly polarized light is employed as a probe light to estimate the corresponding DOP of the current environment, and the DOP can be obtained according to Eq. (6) by using the measured results of $S_{1}$ and $S_{2}$. Based on the calibrating result of DOP in the current measuring environment, the full Stokes parameters of any incident light can be obtained according to the Eqs. (4) and (5).

The basic working principle and measuring procedure of the proposed scheme will be introduced as follows. Firstly, in the PSG system, the transmission axis of the polarizer and the fast 

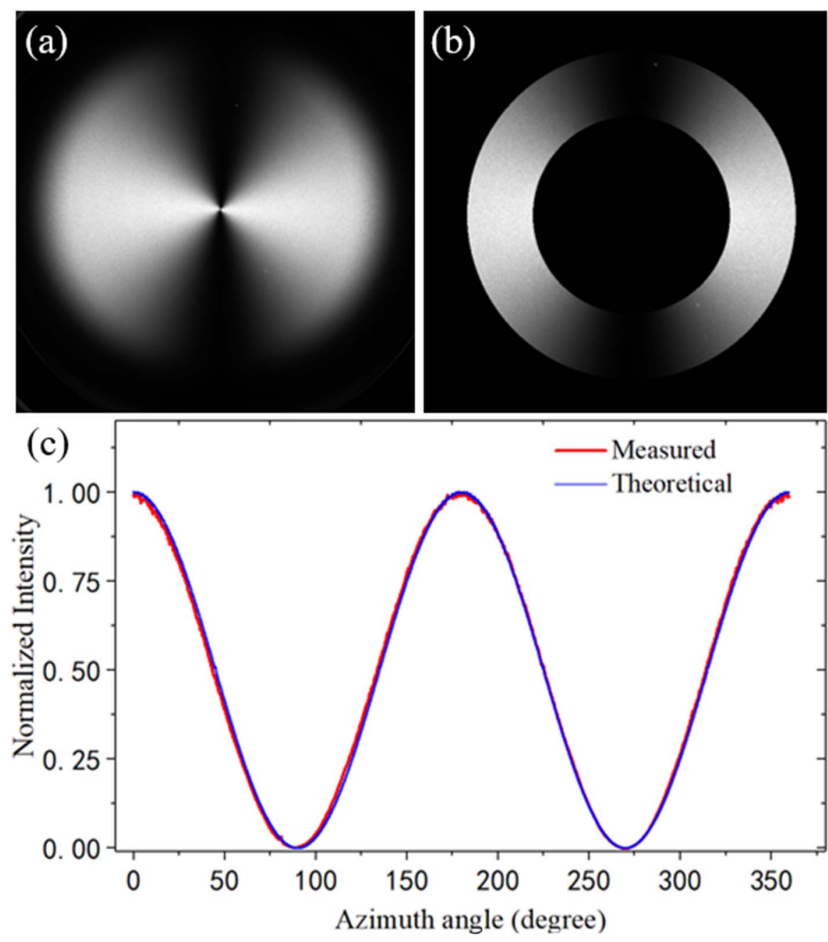

Fig. 2. Typical measured results of linearly polarized light. (a) The recorded hourglass intensity image of horizontal linearly polarized light. (b) A ring ROI cut from (a). (c) The normalized intensity modulation curves as a function of azimuth angle $\varphi$ obtained from measured and theoretical images.

axis of the quarter-wave plate were rotated to $0^{\circ}$ for generating a horizontal linearly polarized input light, which was also employed as the probe light to estimate the DOP. The input light was modulated and detected by the PSD system, the corresponding intensity image was recorded by the camera and shown in Fig. 2(a), and the radius of the hourglass-shaped intensity image is about 800 pixels. As shown in Fig. 2(a), the marginal and central parts were not well modulated, owing to the vignetting effect of the optical system and the imperfection of optical components, so a ring area was cut from Fig. 2(a) and chosen as a region of interest (ROI), which is beneficial for improving the measuring accuracy and image processing speed. To make full use of the modulated information in the image, a thicker ring area is expected, but more defective pixels may be included at the same time, which also limits the measurement accuracy. In our measurement, the ring thickness was chosen as 200 pixels, and its inner and outer radii were 300 pixels and 500 pixels, respectively, which is shown in Fig. 2(b). To obtain the intensity modulation curve $I(\varphi)$, the integrals were performed from the center of the ring image along the radius at each azimuth angle $\varphi$, the integral values at each azimuth angle are glued to form the integral curve, and then the intensity modulation curve $I(\varphi)$ was obtained by normalizing the integral curve. In the real measurement, these procedures were achieved by performing 180 times Radon transformations ${ }^{[32,33]}$ to the top half and the second half of the ring image in Fig. 2(b) from $0^{\circ}$ to $180^{\circ}$ with a step of $2^{\circ}$. The normalized intensity modulation curve was obtained when the minimum distance between the projection line and the origin of the Cartesian coordinate was set to zero, and it is shown in Fig. 2(c) as the red line. For the purpose of comparison, the theoretical intensity modulation curve was also simulated and is shown in Fig. 2(c) as the blue line. It can be clearly found that the measured intensity distribution had a similar tendency and shape with its theoretical curve. Finally, the Stokes parameters of the incident light were obtained by Fourier analysis of the measured intensity modulation curve $I(\varphi)$, and the estimated DOP was 0.9765 in the current measurement situation.

\section{Results and Discussion}

To validate the accuracy and feasibility of our polarimeter, the quantitative polarization measuring experiments have been carried out in two steps. At first, the incident beams with nine particular polarization states have been measured, including the $0^{\circ}$, $45^{\circ}, 90^{\circ}$, and $135^{\circ}$ linearly polarized light. The left and right circularly polarized light and three elliptically polarized lights were generated by the PSG system, and then they were examined by the PSD system, respectively. The measured results have been shown in Table 1.

It can be known from Table 1 that the measured Stokes parameters of the incident light were consistent with their theoretical values, and the maximum measured error (MME) and the average value of the absolute measured error (AVME) for the nine measurements were 0.0385, 0.0333, and 0.0460 (MME of $S_{1}, S_{2}$, and $S_{3}$ ) and 0.0154, 0.0233, and 0.0224 (AVME of $S_{1}$, $S_{2}$, and $S_{3}$ ), respectively, which indicated that the presented scheme is feasible, and its accuracy can meet the needs of general polarization measurement.

To confirm the feasibility and accuracy of our polarimeter further, more experiments have been carried out by changing the polarization state of incident light regularly. In the PSG system, the transmission axis of the polarizer was fixed at $20^{\circ}$, and the fast axis of the quarter-wave plate was rotated from $0^{\circ}$ to $180^{\circ}$ with a step of $5^{\circ}$ to generate 37 different polarization states. The Stokes parameters of the 37 incident beams were measured and plotted in Fig. 3.

According to the experimental results shown in Fig. 3, we can easily find that the measured results were well consistent with the predicted values in both Stokes curves and the Poincare sphere, and the fitted curves based on these discrete experimental results were also well matched with the theoretically predicted results. Moreover, the MME and the AVME for 37 continuous measurements have also been calculated, and the results were $0.0391,0.0319$, and 0.0555 (MME of $S_{1}, S_{2}$, and $S_{3}$ ), 0.0252, 0.0166, and 0.0203 (AVME of $S_{1}, S_{2}$, and $S_{3}$ ), respectively, which further indicated that the proposed scheme is available to measure the polarization state of the arbitrary polarized beam accurately and conveniently.

In summary, 9 discrete measurements with typical SOPs and 37 continuous measurements have been performed successfully to validate the feasibility of our polarimeter, and the results indicated that the measured error range of three Stokes parameters 
Table 1. Measured Results of Incident Beams with Some Particular Polarization States.

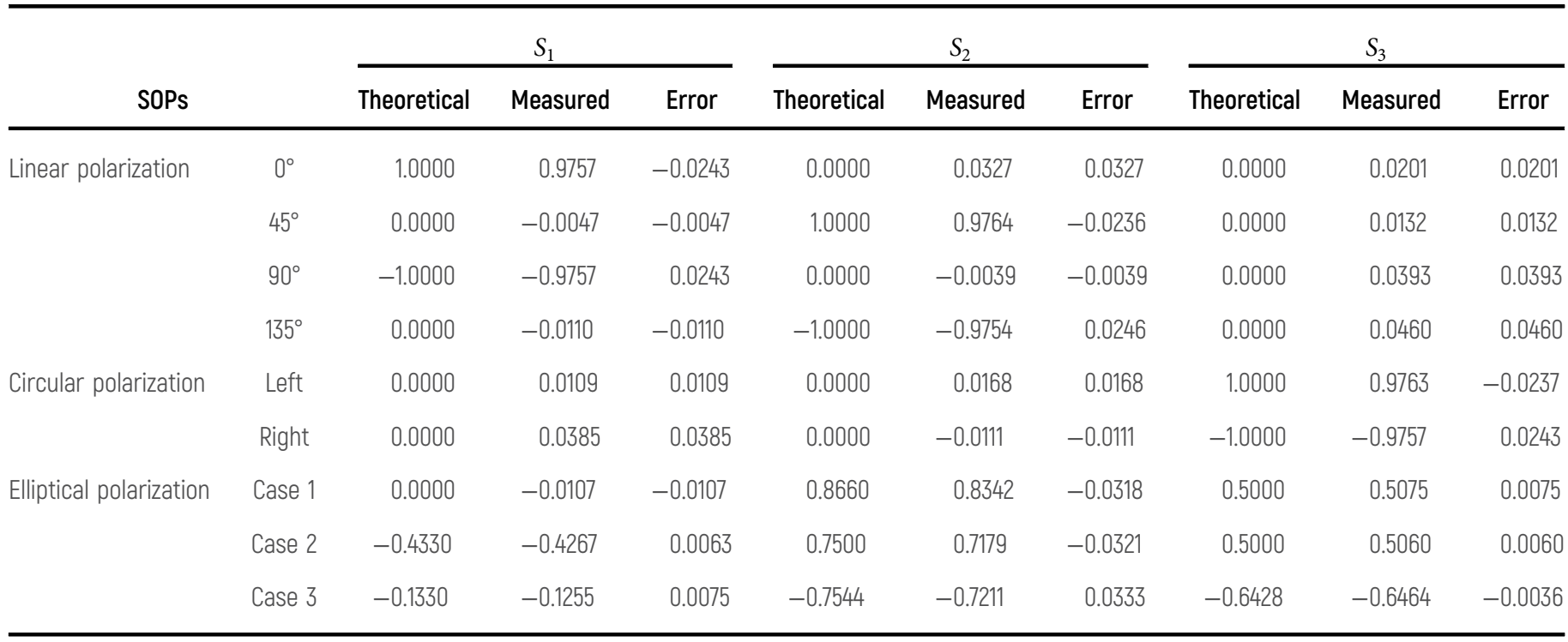

was mainly between 0.01 and 0.05 , which is acceptable in most polarization measurement applications. However, some unavoidable measurement errors still exist, and these errors can probably be attributed to the misalignment of optical components, the imperfections of optical components, the image noise, and ambient stray light. The misalignment of the ZVHR and the analyzer will cause the actual bright areas' orientation to deviate from the ideal position, and this would impair the accuracy of the polarimeter. To improve the accuracy further, the rotating stage with higher accuracy should be utilized in calibration to decrease the influence of misalignment, and better optical components and low noise camera should be employed to improve the modulation quality of the intensity image. Moreover, the whole polarimeter system can be encapsulated in a closed tube to avoid the adverse effect of ambient stray light.

Based on the theoretical analysis and operation procedure of the polarimeter, it is not difficult to conclude that the vortex retarder can be an efficient device for achieving spatial modulation in a polarimeter, and the proposed polarimetry is a novel scheme and easy to implement. This scheme has many distinctive advantages. Firstly, the polarimeter can measure all of the Stokes parameters of polarized light accurately at a single shot, which has potential for fast and precise measurement. Secondly, the configuration of our polarimeter is very simple; it only consists of a vortex retarder, a polarizer, and a camera, which is promising for miniaturization. Thirdly, no mechanical moving part is needed in the polarimeter, and it is very stable and robust. Moreover, the phase retardance change of the ZVHR caused by wavelength drift has little effect on the modulated intensity image, and the SOP can be measured from a single image. Thus, the measurement results are insensitive to the wavelength and power fluctuations of the light source.
However, this scheme still has some unpleasant defects, which are that the current method is only applicable to completely polarized beams, and the handedness of the input light cannot be recognized. Since the fourth Stokes parameter $S_{3}$ is determined by Eq. (5), the estimations of DOP and $S_{3}$ of partially polarized and unpolarized beams will be inaccurate, and the left-handed and right-handed polarized light cannot be distinguished. Fortunately, if a zero-order vortex quarter-wave retarder (ZVQR) is employed to substitute the ZVHR in the PSD system, in a similar way as it is done in a typical Stokes polarimeter using a quarter-wave plate ${ }^{[34]}$, the intensity $I(\varphi)$ can be given as $I(\varphi) \propto S_{0}+\frac{1}{2} S_{1}+\frac{1}{2} S_{1} \cos 2 \varphi+\frac{1}{2} S_{2} \sin 2 \varphi-$ $S_{3} \sin \varphi$. The direct measurement of all of the Stokes parameters can be achieved, and the calibration of the DOP is not needed anymore. Another limitation is that the presented polarimeter is not suitable for spectroscopic measurement because the ZVHR is a wavelength dependent device. To decrease its wavelength dependence, a polarization axis finder (PAF) can be employed to replace the ZVHR and analyzer in the PSD system $^{[31]}$, which provides a potential solution for developing a spectroscopic polarimeter. Moreover, the measurement of the SOPs is based on the Fourier analysis of the hourglass-shaped intensity image, and a well-modulated intensity image is very important for obtaining accurate measurement results; thus, the incident light field should have a homogeneous polarization state and nearly flat intensity distribution all over the captured region. It is also regrettable that the current image processing procedure is relatively slow, because the intensity modulation curve $I(\varphi)$ as a function of azimuth angle $\varphi$ was obtained by performing a series of Radon transformations around the ring image. However, the measurement time can be decreased remarkably if a more advanced image processing algorithm is developed in our next version. 

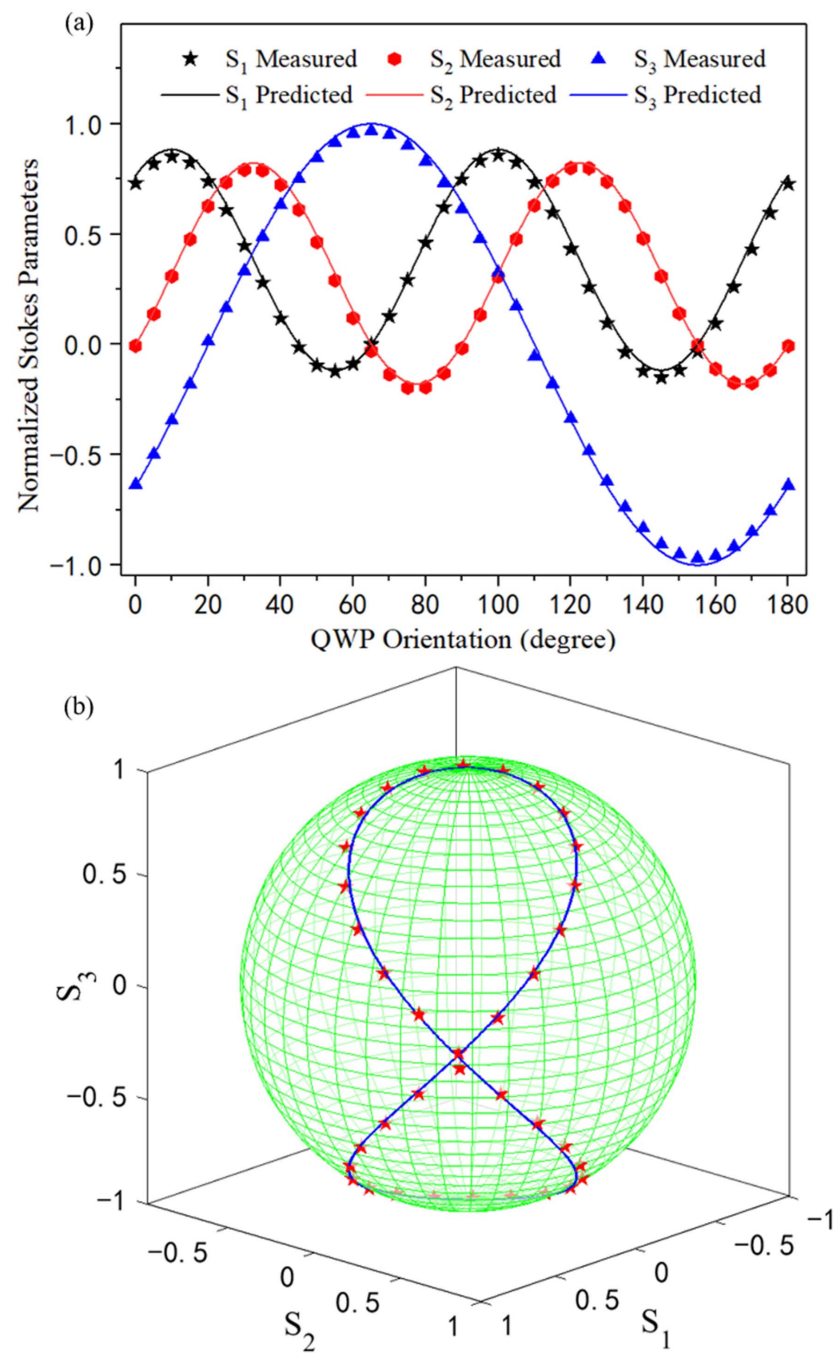

Fig. 3. Experimental results measured from multiple regularly varied SOPS. (a) Experimental (black star, $S_{1}$; red circle, $S_{2}$; blue triangle, $S_{3}$ ) and theoretical (black line, $S_{;}$red line, $S_{2} ;$ blue line, $S_{3}$ ) Stokes parameters corresponding to different SOPs. (b) Experimental (red star) and theoretical (blue line) data shown on a Poincaré sphere.

\section{Conclusion}

In conclusion, a spatially modulated polarimetry scheme has been demonstrated based on the vortex retarder and spatial Fourier analysis. In a single acquisition of the spatially modulated image, the four Stokes parameters of input light can be measured, and the experimental results indicate that this approach is accurate, feasible, and convenient for examining the polarization states of light.

\section{Acknowledgement}

This work was supported by the National Natural Science Foundation of China (NSFC) (No. 61975235) and the Natural Science Foundation of Hunan Province (No. 2019JJ40342).

\section{References}

1. H. Fujiwara, Spectroscopic Ellipsometry: Principles and Applications (Wiley, 2007).

2. Z. Sun, Y. Huang, Y. Bao, and D. Wu, "Polarized remote sensing: a note on the Stokes parameters measurements from natural and man-made targets using a spectrometer," IEEE Trans. Geosci. Remote 55, 4008 (2017).

3. Y. Feng, L. Li, J. Lin, H. Xu, W. Zhang, X. Tang, L. Xi, and X. Zhang, "Joint tracking and equalization scheme for multi-polarization effects in coherent optical communication systems," Opt. Express 24, 25491 (2016).

4. Y. Cao, X. Liu, Z. Xia, W. Wang, N. P. Yadav, and W. Liu, "Sensing of ultrasonic fields based on polarization parametric indirect microscopic imaging," Chin. Opt. Lett. 17, 051601 (2019).

5. J. Li, Y. Li, K. Liu, G. Zhou, L. Liu, and M. Zheng, "Hybrid calibration method of a wide-view-angle Mueller polarimeter for hyper-numerical-aperture imaging systems," Chin. Opt. Lett. 18, 081202 (2020).

6. H. Dai and C. Yan, "Measurement errors resulted from misalignment errors of the retarder in a rotating-retarder complete Stokes polarimeter," Opt. Express 22, 11869 (2014).

7. R. M. Azzam, "Light polarization: a rich source of information," Opt. Acta 29, 685 (1982).

8. S. Jiang, H. Jia, Y. Lei, X. Shen, J. Cao, and N. Wang, "Novel method for determination of optical rotatory dispersion spectrum by using line scan CCD," Opt. Express 25, 7445 (2017).

9. G. Vishnyakov, G. Levin, and A. Lomakin, "Measuring the angle of rotation of the plane of polarization by differential polarimetry with a rotating analyzer," J. Opt. Technol. 78, 124 (2011).

10. A. Álvarez-Herrero, P. G. Parejo, and M. Silva-López, "Fine tuning method for optimization of liquid crystal based polarimeters," Opt. Express 26, 12038 (2018).

11. W. A. Woźniak, M. Prętka, and P. Kurzynowski, "Imaging Stokes polarimeter based on a single liquid crystal variable retarder," Appl. Opt. 54, 6177 (2015).

12. G.-P. Lemus-Alonso, C. Meneses-Fabian, and R. Kantun-Montiel, “One-shot carrier fringe polarimeter in a double-aperture common-path interferometer," Opt. Express 26, 17624 (2018).

13. Q. Liu, C. Bai, J. Liu, J. He, and J. Li, "Fourier transform imaging spectropolarimeter using ferroelectric liquid crystals and Wollaston interferometer," Opt. Express 25, 19904 (2017).

14. M. Mujat, E. Baleine, and A. Dogariu, "Interferometric imaging polarimeter," J. Opt. Soc. Am. A 21, 2244 (2004).

15. X. Zhang, S. Yang, W. Yue, Q. Xu, C. Tian, X. Zhang, E. Plum, S. Zhang, J. Han, and W. Zhang, "Direct polarization measurement using a multiplexed Pancharatnam-Berry metahologram," Optica 6, 1190 (2019).

16. S. Hermon, A. Ma, F. Yue, F. Kubrom, Y. Intaravanne, J. Han, Y. Ma, and $\mathrm{X}$. Chen, "Metasurface hologram for polarization measurement," Opt. Lett. 44, 4436 (2019).

17. A. Pors, M. G. Nielsen, and S. I. Bozhevolnyi, "Plasmonic metagratings for simultaneous determination of Stokes parameters," Optica 2, 716 (2015).

18. J. Zhang, C. Yuan, G. Huang, Y. Zhao, W. Ren, Q. Cao, J. Li, and M. Jin, "Acquisition of a full-resolution image and aliasing reduction for a spatially modulated imaging polarimeter with two snapshots," Appl. Opt. 57, 2376 (2018).

19. J. Chang, N. Zeng, H. He, Y. He, and H. Ma, "Single-shot spatially modulated Stokes polarimeter based on a GRIN lens," Opt. Lett. 39, 2656 (2014).

20. M. W. Kudenov, M. J. Escuti, E. L. Dereniak, and K. Oka, "White-light channeled imaging polarimeter using broadband polarization gratings," Appl. Opt. 50, 2283 (2011).

21. G. Biener, A. Niv, V. Kleiner, and E. Hasman, "Near-field Fourier transform polarimetry by use of a discrete space-variant subwavelength grating," J. Opt. Soc. Am. A 20, 1940 (2003).

22. Z. E. Bomzon, G. Biener, V. Kleiner, and E. Hasman, "Spatial Fourier-transform polarimetry using space-variant subwavelength metal-stripe polarizers," Opt. Lett. 26, 1711 (2001).

23. Q. Cao, J. Zhang, E. DeHoog, and C. Zhang, "Demonstration of snapshot imaging polarimeter using modified Savart polariscopes," Appl. Opt. 55, 954 (2016).

24. Y.-L. Lo, J.-F. Lin, and S.-Y. Lee, "Polariscope for simultaneous measurement of the principal axis and the phase retardation by use of two phase-locked extractions," Appl. Opt. 43, 6248 (2004). 
25. W. A. Woźniak and P. Kurzynowski, "Compact spatial polariscope for light polarization state analysis," Opt. Express 16, 10471 (2008).

26. K. Oka and T. Kaneko, "Compact complete imaging polarimeter using birefringent wedge prisms," Opt. Express 11, 1510 (2003).

27. S. C. McEldowney, D. M. Shemo, R. A. Chipman, and P. K. Smith, "Creating vortex retarders using photoaligned liquid crystal polymers," Opt. Lett. 33, 134 (2008).

28. Z. He, Y. Zheng, H. Liu, M. Li, H. Lu, H. Zhang, Q. Feng, and D. Mao, "Passively $Q$-switched cylindrical vector laser based on a black phosphorus saturable absorber," Chin. Opt. Lett. 17, 020004 (2019).

29. J. Chen, C. Wan, and Q. Zhan, "Vectorial optical fields: recent advances and future prospects," Sci. Bull. 63, 54 (2018).
30. C. Gao and B. Lei, "Spatially polarization-modulated ellipsometry based on the vectorial optical field and image processing," Appl. Opt. 59, 5377 (2020).

31. B. Lei and S. Liu, "Efficient polarization direction measurement by utilizing the polarization axis finder and digital image processing," Opt. Lett. 43, 2969 (2018).

32. R. Deans, The Radon Transform and Some of Its Applications (Courier, 2007).

33. W. Zhang, X. Zhang, Y. Cao, H. Liu, and Z. Liu, "Robust sky light polarization detection with an S-wave plate in a light field camera," Appl. Opt. 55, 3518 (2016).

34. D. H. Goldstein, Polarized Light (CRC Press, 2011). 